\title{
ANALYSIS OF STUDENTS' SELF-ASSESSMENT AND GENERIC SKILLS USING DAIFUKU-CHO AND PROG TEST
}

\author{
Akiko Takahashi, Yosuke Tohata, Hideyuki Kobayashi, \\ Yoshiaki Rikitake and Yoshikatsu Kubota \\ National Institute of Technology (KOSEN), Sendai College, Sendai, Miyagi, Japan
}

\begin{abstract}
The present study analyzes the relationship between a teacher's evaluation of students' self-assessment by "Daifuku-cho," which is used for teacher-and-student interaction in PE class, and their competency improvement using PROG test scores. It turns out that there is a correlation between them, and the higher the teacher's evaluation of students' comments is, the higher the students' scores in "Competency" part of PROG test are. Compared with athletic skills, competency improvement in PE class is difficult to assess, but this study suggests that by evaluating students' self-assessment in "Daifuku-cho," we could possibly estimate their competency improvement.
\end{abstract}

\section{KEYWORDS}

Self-Assessment, Generic Skills, Daifuku-cho, PROG Test

\section{INTRODUCTION}

School education in Japan is rapidly transforming today. One-way lecture-based style used to be the teaching norm, in which teachers one-sidedly give students a lot of information and knowledge of academic subjects. Today active-learning style education is flourishing, in which students collaboratively find problems and try to solve them. To encourage students learn actively, we have carried out Active Learning (AL) [Niemi 2002, Hassan 2015], Mastery Learning (ML) [Kularbphettonga 2015, Shafie 2010, Kulik 1990], and Problem/Project Based Learning (PBL) [Rodríguez 2015, Fernandes 2014] and tried to foster students' literacy and competency at Institute of Technology (KOSEN), Sendai College (Sendai KOSEN). We also have evaluated students' achievements in various measures. [Takahashi 2016, Kubota 2017]

Although evaluation of students' academic achievements so far have been based on examination scores which assess their mastery of teaching contents and also their literacy and competency test scores, students themselves have been unable to assess what they are learning and how much they are growing in their abilities. Therefore, this evaluation method is not adequate for students to work on continuous self-assessment. Therefore, we need a framework to encourage students to assess their own daily growth and enhance their learning continuously.

The present study especially focuses on students' competency, and discusses how students' own review and reflection, or self-assessment, and teachers' feedback promote the students' personal growth, comparing individual student's growth using the annual test results of competency evaluation. We report that the students' reflective use of "Daifuku-cho," a kind of shuttle card between teachers and students which contains records of students' learning and teacher's comments on them, enhances their learning [Kogo 2006, Kogo 2007]. By comparing the contents of "Daifuku-cho" and grades and the results of PROG test, we suggest that the better students reflect themselves using "Daifuku-cho," the better their competency becomes. 


\section{RELATED WORKS}

In Japan, Cabinet Office is asking colleges and universities to develop human resources with high skills which full-fledged members of society are required to have. Ministry of Education, Culture, Sports, Science and Technology, Japan (MEXT) is also promoting the introduction of AL into Japanese schools and colleges to cultivate human resources who can think and act on their own [MEXT 2014]. Quite a few number of high schools and universities all over Japan are now working on AL.

As for the skills members of society are required to have, in UK, the three basic skills, which comprise a national Key Skills Qualification, include that communication, numeracy or the application of numbers and use of information technology. The three wider key skills are working with others, improving own learning and performance and problem-solving [Confederation of British Industry 1998], and they are called core skills. In Canada, they are called Employability Skill [Conference Board of Canada], and are categorized into Fundamental Skills, Personal Management Skills and Teamwork Skills.

OECD is also analyzing the results of various international surveys and existing studies, and abilities of students and adults. PISA Surveys investigate literacy, how people can make use of knowledge they acquired [OECD 2003], and DeSeCo is working on research about abilities and notions of foundation of sustainability as key competencies [OECD 2005]. In Hong Kong, the new Hong Kong three-year senior secondary curriculum (NSS) is aiming to improve students' abilities to think critically and creatively. Ka-Cheng and et al. are trying to improve The Hong Kong Diploma of Secondary Education (HKDSE) Examination, the official evaluation approach, since it is inadequate to measure Generic skills [Leung 2014].

PROG test is used in many Japanese educational institutes for quantitative assessment and it is used to evaluate students' competencies compared with those university graduates are required to have [Kawaijuku]. Ito et al. report that university students' Grade Point Average (GPA) and PROG have weak correlation [Ito 2014]. Fujiki et al. examine the effectiveness of "Fundamental Competencies for Working Persons" advocated by Ministry of Economy, Trade and Industry, Japan, in KOSEN's first-year education using PROG test [Fujiki 2016]. As described above, attempts to improve generic skills and to measure their effectiveness have been carried out all around the world.

The use of shuttle-card "Daifuku-cho," which is used by students to review and reflect classes and to communicate with teachers, was proposed [Oda 1991]. "Daifuku-cho" has columns for students to fill in and ones for teachers to write some comments for students. After each class, students write what they felt in class or some questions in a column and submit it to the teacher. The teacher in return writes some comments and returns it to the students in the next class. "Daifuku-cho" enables teachers and students to interact, and students can review what they learned in the class and learn from the teacher's comments. Kogo reports that the communication between students and teachers through "Daifuku-cho" leads to the students' introspective review of the class and higher class attendance rates. Moreover, he reports the use of "Daifuku-cho" in e-learning courses where face-to-face communication is hard and verifies that it contributes to reducing the distance between students and teachers. "Daifuku-cho" proves effective to encourage students to look back on their daily learning.

As described above, a lot of methods of evaluating competency and of improving learning outcomes by students' daily reflection have been proposed. In the present study we take physical education (PE) class as a test bed, in which we can see little difference among students' abilities of comprehension, and examine the relationship between the quantitative evaluation method and effectiveness of students' daily reflection of learning.

\section{EVALUATION BY CORRELATION BETWEEN PROG TEST AND GRADES BASED ON "DAIFUKU-CHO"}

\subsection{Outline of Evaluation Using PROG test and "Daifukucho"}

In the present study, we conducted a follow-up review of 2nd-year students (16 years old) and 4th-year students (19 years old) using PROG test and "Daifuku-cho." PROG test evaluates both "Literacy" and 
"Competency," and in this study we use "Competency" such as "ability to deal with environment surrounding in a practical manner" or "personal traits like policy of decision-making and action to cope with their surroundings" as one evaluation index, because it is more important than "Literacy" in "Fundamental Competencies for Working Persons." We used "Daifuku-cho" shown in Figure 1 as the other evaluation index and focused on PE class, in which each student's understanding does not lead to their grades straightforward. In the present study, we will show the effectiveness of "Daifuku-cho" as a tool for students to review their daily learning by examining the correlation of these two indices.

\subsection{Structure of "Daifuku-cho}

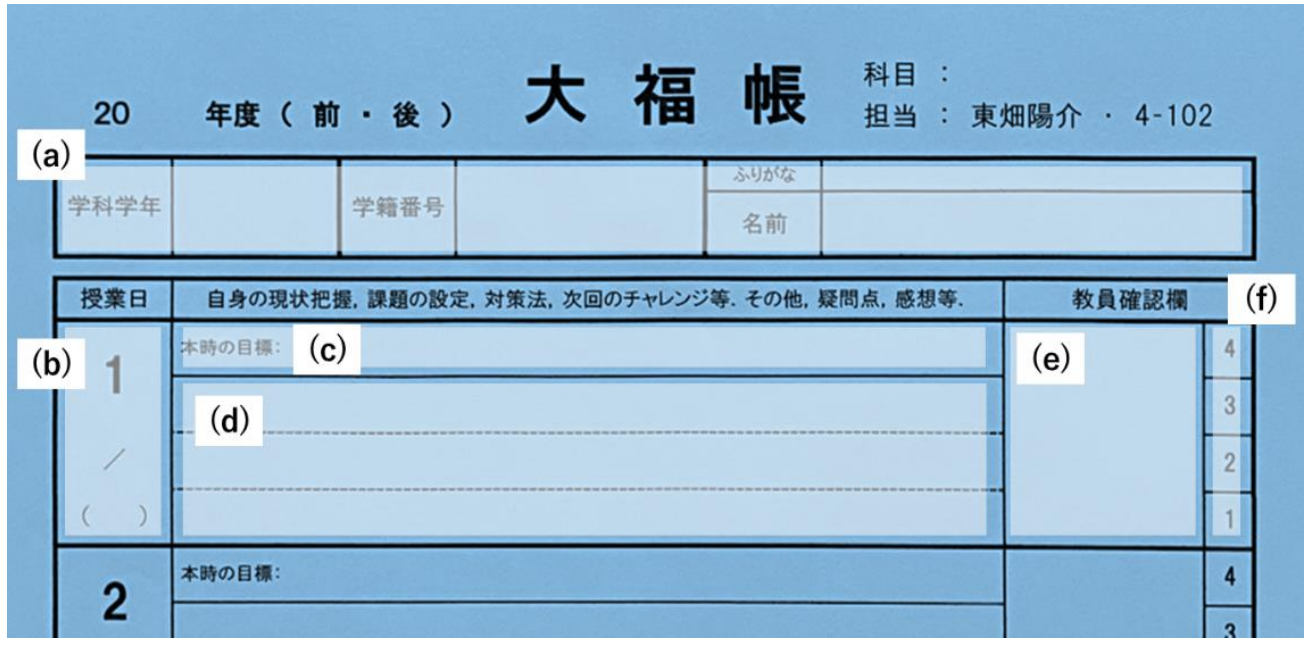

Figure 1. Structure of "Daifuku-cho" utilized in PE class

"Daifuku-cho" is a double-sided, A4-size thick sheet of paper and 15 columns for one semester are printed on it. As shown in Figure 1, it consists of parts (a)-(f).

(a) Student ID: grade, class, student number, and name

(b) Class number and Date: the date of the class

(c) Goal of class: Students describe their personal goal of the class, what and how they are going to work on in the class, how they are going to improve themselves and contribute to the class, and so on.

(d) Review of class: The most important part of "Daifuku-cho." In terms of their own goal and class contents, students write:

- How they understand the present state they are in and identify the problems: how far they have achieved or have not achieved.

- Setting up the tasks to achieve their goals: What factors are helping their achievement or preventing them, and what tasks are needed to solve the problems they are facing.

- Reflecting and describing their practices and challenges: To overcome the factors which preventing their achievement, what resources and methods they can try. Questions to the teacher or comments on the class.

(e) Teacher's feedback to what the student wrote. Adding special knowledge or answering the student's questions.

(f) Assessment: Teacher assesses what the student wrote in (d), whether they are helpful to take concrete steps to achieve their goals, whether they think logically and so on, on a scale of one to four and the full marks in one semester are 60. 


\subsection{Comparison Between the Results of PROG test and the Evaluation by} "Daifuku-cho"

Table 1. Each student's PROG competency and grade in Daifuku-cho

\begin{tabular}{|c|c|c|c|c|}
\hline Student ID & $\begin{array}{l}2^{\text {nd }} \text { grade: } \\
\text { Competency }\end{array}$ & $\begin{array}{c}4^{\text {th }} \text { grade: } \\
\text { Competency }\end{array}$ & $\begin{array}{l}2^{\text {nd }} \text { grade: } \\
\text { Daifuku-cho }\end{array}$ & $\begin{array}{c}4^{\text {th }} \text { grade: } \\
\text { Daifuku-cho }\end{array}$ \\
\hline 1 & 5 & 6 & 0.89 & 0.92 \\
\hline 2 & 5 & 6 & 0.89 & 0.93 \\
\hline 3 & 5 & 6 & 0.50 & 0.92 \\
\hline 4 & 2 & 4 & 0.93 & 0.85 \\
\hline 5 & 5 & 6 & 0.79 & 0.83 \\
\hline 6 & 4 & 5 & 0.89 & 0.97 \\
\hline 7 & 5 & 6 & 0.93 & 0.83 \\
\hline 8 & 2 & 3 & 0.96 & 0.88 \\
\hline 9 & 1 & 7 & 0.89 & 0.88 \\
\hline 10 & 4 & 6 & 0.93 & 1.00 \\
\hline 11 & 4 & 5 & 0.68 & 0.77 \\
\hline 12 & 3 & 6 & 0.75 & 0.95 \\
\hline 13 & 1 & 1 & 0.86 & 0.85 \\
\hline 14 & 1 & 1 & 0.61 & 0.68 \\
\hline 15 & 3 & 3 & 0.82 & 0.85 \\
\hline 16 & 4 & 4 & 0.93 & 1.00 \\
\hline 17 & 3 & 3 & 0.93 & 0.83 \\
\hline 18 & 4 & 4 & 0.86 & 0.85 \\
\hline 19 & 1 & 1 & 0.89 & 0.80 \\
\hline 20 & 3 & 3 & 0.86 & 0.88 \\
\hline 21 & 1 & 1 & 0.75 & 0.78 \\
\hline 22 & 1 & 1 & 0.89 & 0.87 \\
\hline 23 & 1 & 1 & 0.86 & 0.85 \\
\hline 24 & 6 & 2 & 0.79 & 0.82 \\
\hline 25 & 4 & 3 & 0.86 & 0.88 \\
\hline 26 & 2 & 1 & 0.86 & 0.83 \\
\hline 27 & 5 & 4 & 0.93 & 0.82 \\
\hline 28 & 5 & 4 & 1.00 & 0.98 \\
\hline 29 & 4 & 1 & 0.93 & 0.92 \\
\hline 30 & 4 & 3 & 0.89 & 0.93 \\
\hline 31 & 6 & 5 & 0.89 & 0.80 \\
\hline 32 & 3 & 2 & 0.96 & 0.90 \\
\hline
\end{tabular}




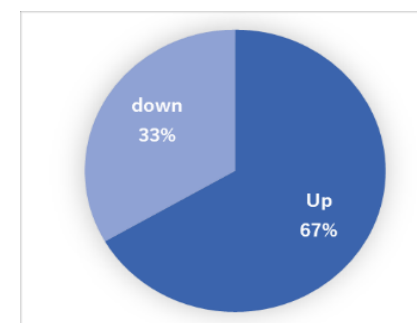

(a) Up

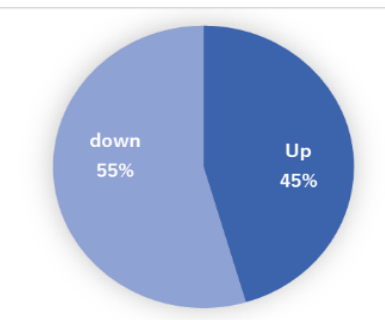

(b) No change

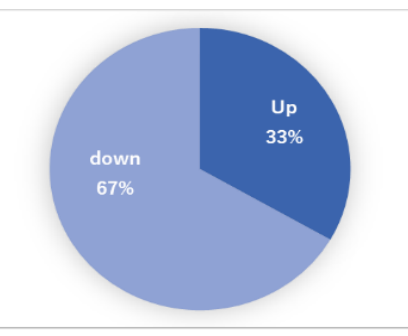

(c) Down

Figure 2. Relationship between PROG competency and grade in Daifuku-cho

Table 1 shows the results of PROG test and scores of "Daifuku-cho" of students in one class when they were in the $2^{\text {nd }}$ grade and in the $4^{\text {th }}$ grade. The students from 1 to 12 show the improvement of their competency in two years from their 2 nd grade to 4 th grade (Up group). The students from 13 to 23 do not show any change in their competency (No change group). The students from 24 to 32 show drop in their competency scores (Down group).

Out of the 12 students in the Up group, eight students got better in "Daifukucho" assessment, that is, 67\% of the students got better, but four did not change. Five students out of 11 No Change group members got better, which means $45 \%$ of them, but the other six dropped in "Daifuku-cho." Three out of nine of the Down group, which is $33 \%$ of them, but the other six dropped. Figure 2 showus the percentages in each group. The percentage of students who got better is the highest in (a), and the lowest in (c).

Figure 3 shows the amount of change in students' grades of each group between their $2^{\text {nd }}$ year and $4^{\text {th }}$ year in box-and-whisker plot. The boxes show interquartile range (Q25-Q75) of each group, the bold lines the Median, the upper whiskers Max, the lower whiskers Min, and the dots indicate outliers. As it shows, the Up group got better in the average grade. The result of Tukey-Kramer test shows that there are significant differences among the average scores of each group using a level of significance of $\mathrm{p}<.05$.

Therefore, those who improved in their competency showed the tendency to get better in "Daifuku-cho" scores, while those who decreased in their competency scores had the tendency to go down in the average scores.

\subsection{Discussion}

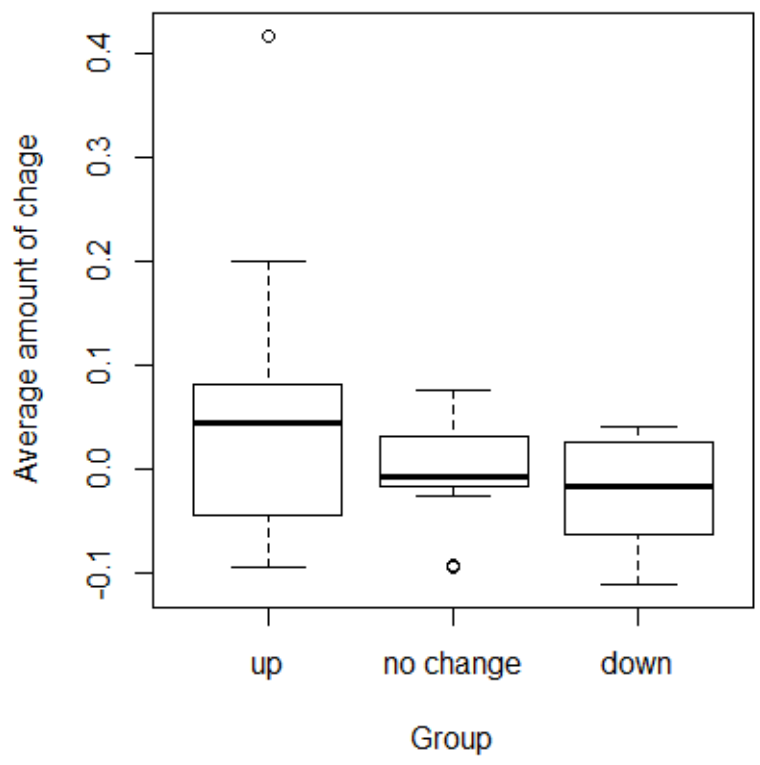

Figure 3. Competency and improvement in grade 
The results of the present study indicate that the better the students get in their competency, the better their grades become, and vice versa.

We focused on the relationship of "Daifuku-cho" scores and "Competency" in PROG test, and assessed students' grades in PE class. Assessing students' athletic skills is a regular part of PE, but on the other hand, it is relatively difficult to assess students' cognitive skills in PE class. We, however, were able to show the possibility of assessing the improvement in students' competency through the use of "Daifuku-cho."

Ito et al. shows that there is a weak correlation between GPA and PROG [Ito 2014]. In the present study, we also found out that there was a weak correlation between PROG and PE class grades, which suggests that we can assess the improvement in students' competency through the assessment by "Daifuku-cho."

So far, "Daifuku-cho" has been used as a communication tool between teachers and students, and also as a reflective tool for students. Our present study shows another potential of "Daifuku-cho" as a tool to measure the improvement in students' competency.

\section{CONCLUSION}

"Daifuku-cho" is used as a tool for students' own review of daily learning and teachers' feedback. The present study aims to investigate the learning effects by "Daifuku-cho" in terms of individual student's growth in competency.

We conducted a two-year follow-up review of 32 students and examined the correlation of grading by "Daifuku-cho" and the quantitative evaluation of their competency using PROG test. The students were divided into three groups: those whose competency scores improved in two years, those whose competency scores showed no change, and those whose competency scores dropped. There were significant differences among the average scores of "Daifuku-cho" of each group and those whose competency scores improved also showed improvement in their "Daifuku-cho" scores. It suggests a possibility that we could estimate a student's competency improvement according to the evaluation of his/her "Daifuku-cho."

The present study shows that there is a correlation between the evaluation of students' "Daifuku-cho" and their competency improvement. Among future issues, we need to analyze the scores of each group qualitatively based on the entries to "Daifuku-cho." By clarifying the features of entries to "Daifuku-cho" in terms of competency improvement, we hope to show some new evaluation points of "Daifuku-cho," which may lead to "Fundamental Competencies for Working Persons."

\section{ACKNOWLEDGEMENT}

This research was partially funded through the financial assistance of The Ministry of Education, Culture, Sports, Science and Technology Acceleration Program for University Education Rebuilding.

\section{REFERENCES}

Confederation of British Industry, 1998. "Qualified to compete Creating a world class qualifications framework," Human Resources Brief, January, London: CBI.

Conference Board of Canada, "Employability Skills," https://www.conferenceboard.ca/edu/employability-skills.aspx

Fernandes, S.R.G., 2014. 'Preparing graduates for professional practice: findings from a case study of Project-based Learning (PBL),' Procedia - Social and Behavioral Sciences, Vol. 139, pp. 219-226.

Fujiki M.N. et al, 2016. "The practical program for bringing out generic skills of the first year students," TALE, pp. 271-277, Dec., 2016.

Hassan, N.F. et al, 2015. 'Student understanding through the application of technology enabled Active Learning in practical training,' Procedia - Social and Behavioral Sciences, Vol. 204, pp. 318-325.

Ito H., 2014. "Assessing an Assessment Tool of Higher Education: Progress Report on Generic Skills (PROG) in Japan," IJERE, Vol.3, No.1, pp.1-10.

Kawaijuku and Riasec, "PROG: Progress report on generic skills," https://www.kawaijuku.jp/jp/research/prog/point.html Kogo C., 2006. "What Aspect of Class Did Shuttle Card “Daifukucho” Change?,” JSET06-5, pp.23 —30. 
Kogo C., 2007. "Using Shuttle Card "Daifuku-cho" in e-Learning Courses," JSET07-05, pp. 297-300.

Kubota, Y. et al, 2017. "Analysis of Active Learning Suitability of Subjects in Information and Electronics," International Journal: Engineering Pedagogy, Vol.7, No.3, pp. 19-33.

Kularbphettonga K. et al, 2015. 'Developing an adaptive Web-Based Intelligent Tutoring System using Mastery Learning technique,' Procedia - Social and Behavioral Sciences, Vol. 191, pp. 686-691.

Kulik, C.L.C. et al, 1990. 'Effectiveness of Mastery Learning Programs: A Meta-Analysis,' REVIEW OF EDUCA'TIONAL RESEARCH Summer, Vol. 60 no.2 265-299.

Leung K. et al, 2014, "A study of the alignment of learning targets and assessment to generic skills in the new senior secondary mathematics curriculum in Hong Kong," Studies in Educational Evaluation, No. 43, pp.115-132.

MEXT, 2014, '2014 White Paper on Education, Culture, Sports, Science and Technology,' Ministry of Education, Culture, Sports, Science and Technology-JAPAN, http://www.mext.go.jp/b_menu/hakusho/html/hpab201401/1376911.htm

Niemi, H., 2002. 'Active learning - a cultural change needed in teacher education and schools,' Teaching and Teacher Education, vol. 18, pp. 763-780.

OECD, 2003. "Learning for Tomorrow's World, First Results from PISA." OECD.

OECD, 2005. "The Definition and Selection of Key Competencies (DeSeCo)," OECD.

Oda K., 1991. "Teaching Practice on the Effects of Shuttle-Card "Daifuku", Bulletin of Faculty of Education, Mie University Educational Science Educational Science (42), p165-174. (in Japanese)

Rodri'guez, J. et al, 2015. 'Project Based Learning experiences in the space engineering education at Technical University of Madrid,' Advances in Space Research, Vol. 56, pp. 1319-1330.

Shafie, N., et al, 2010. 'Mastery Learning Assessment Model (MLAM) in teaching and learning mathematics,' Procedia Social and Behavioral Sciences, Vol. 8, pp. 294-298.

Takahashi, A. et al, 2016. "A3 Learning System: Advanced Active and Autonomous Learning System,” International Journal of Engineering Pedagogy, Vol.6, Issue2, pp. 52-58. 